\title{
Triggered release from lipid bilayer vesicles by an artificial transmembrane signal transduction system
}

\author{
Matthew J. Langton, ${ }^{\mathrm{a}}$ Lorel M. Scriven, ${ }^{\mathrm{a}}$ Nicholas H. Williams ${ }^{\mathrm{b}^{*}}$ and Christopher A. Hunter ${ }^{{ }^{*}}$ \\ ${ }^{a}$ Department of Chemistry, University of Cambridge, Lensfield Road, Cambridge CB2 1EW, United Kingdom

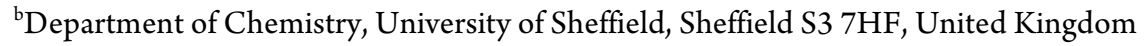 \\ KEYWORDS Signal transduction; Triggered release, Vesicles, Bilayer membranes; Liposomes; Molecular motion; Switchable catalyst.
}

\begin{abstract}
The on-demand delivery of drug molecules from nano-scale carriers with spatio-temporal control is a key challenge in modern medicine. Here we show that lipid bilayer vesicles (liposomes) can be triggered to release an encapsulated molecular cargo in response to an external control signal by employing an artificial transmembrane signal transduction mechanism. A synthetic signal transducer embedded in the lipid bilayer membrane acts as a switchable catalyst, catalyzing the formation of surfactant molecules inside the vesicle in response to a change in external $\mathrm{pH}$. The surfactant permeabilises the lipid bilayer membrane to facilitate release of an encapsulated hydrophilic cargo. In the absence of the $\mathrm{pH}$ control signal, the catalyst is inactive and the cargo remains encapsulated within the vesicle.
\end{abstract}

\section{INTRODUCTION}

Transmembrane signal transduction and signal amplification are important features of numerous biological processes, facilitating the communication of chemical information across lipid bilayer membranes. $^{1}$ In Nature, signalling is achieved by membrane-spanning proteins, such as G-protein coupled receptors, ${ }^{2}$ that generate an intra-cellular signal (second messenger) in response to binding of an extra-cellular signalling molecule (first messenger). Second messengers trigger signalling cascades that lead to the cellular response. Typically, such cascades also amplify the signal by initiating enzyme catalysed processes. Many synthetic supramolecular systems capable of physically transporting chemical signals, such as ions, across lipid bilayer membranes have been reported..$^{3-9}$ However, mimicking signal transduction and amplification processes that occur without physical transfer of matter has proved a considerable challenge for synthetic systems. ${ }^{10-15}$ Recently, we have shown that a new abiotic mechanism - membrane translocation - can be used for signal transduction across lipid bilayers in artificial systems (Figure 1a). The reversible movement of a synthetic transducer molecule across a bilayer can be controlled by chemical input signals on the outside of the vesicle. The position of the transducer is coupled to catalyst activation, so that an amplified output signal is generated on the inside of the vesicle. ${ }^{16,17}$

One outcome of biological transmembrane signal transduction can be the activation of channels that control the influx or efflux of ions, such as calcium, in response to extra-cellular signals. ${ }^{1,18}$ We hypothesized that a transmembrane signalling process could be used in a similar way to trigger release of membrane-impermeable solutes from inside vesicles. Lipid bilayer vesicles are attractive candidates for the delivery of a range of therapeutics, ${ }^{19,20}$ and there are established clinical applications in the delivery of anticancer agents. ${ }^{21,22}$
In recent years, significant research effort has been applied to developing responsive liposomes, where the release of a payload is triggered in response to an external or environmental stimulus (e.g. $\mathrm{pH}$, enzymes and temperature). ${ }^{23}$ Here we show that an artificial signal transduction mechanism can be used to control the formation of surfactants inside lipid bilayer vesicles, triggering the release of an internal cargo in response to a change in external $\mathrm{pH}$.

\section{APPROACH}

The addition of amphiphilic surfactants to lipid bilayer vesicles leads to lysis by solubilisation of the lipids. ${ }^{24-26}$ However at subsolubilising concentrations, surfactants also have profound effects on the properties of lipid bilayers, leading to curvature strain, alkyl chain disordering, lateral expansion and increased permeability to hydrophilic solutes without destruction of the vesicle. ${ }^{27}$ To exploit this effect, we employed an artificial transmembrane signal transducer to initiate the production of low concentrations of surfactant inside lipid bilayer vesicles (Fig. 1b). The controlled generation of surfactant provides a mechanism for the release of encapsulated cargo by permeabilising the lipid bilayer (Fig. 1c). Whilst the synthesis of surfactant molecules in bilayer systems has been explored in the context of self-replicating fatty acid vesicles, in which the (auto)catalytic formation of fatty acids from precursors leads to self-reproduction of the vesicles, ${ }^{28}$ the controlled production of surfactants to release a cargo from lipid bilayer vesicles has not been explored.

The triggered release process is shown schematically in Figure 1. A high concentration of the water-soluble cargo (pink) and a low concentration of the hydrophilic surfactant precursor (grey) are encapsulated in vesicles that have a synthetic signal transducer embedded in the membrane. 


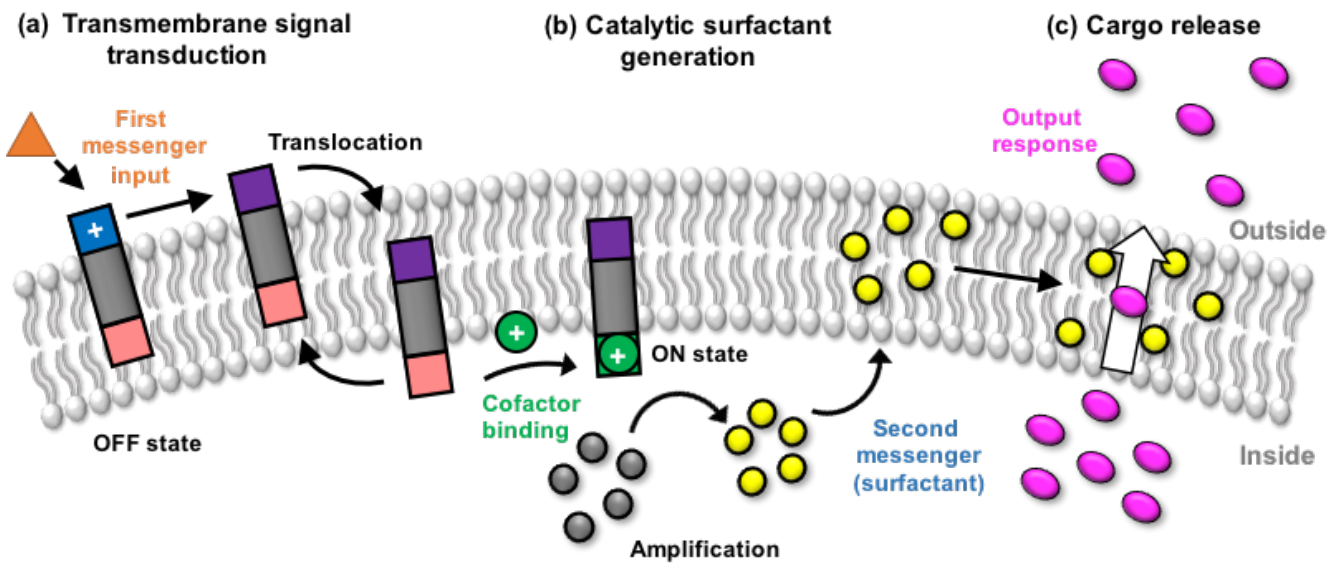

(b) Catalytic surfactant (c) Cargo release (d) Synthetic transducer

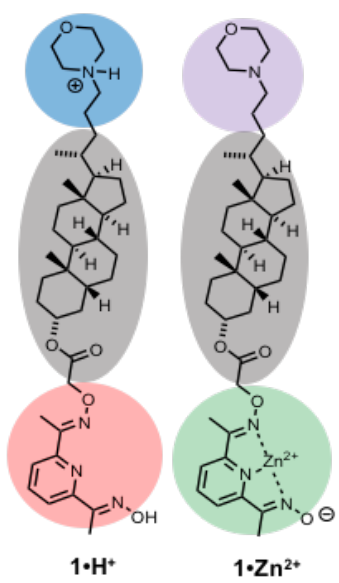

Figure 1. Triggered cargo release from vesicles using an artificial signal transduction mechanism. (a) Transmembrane signal transduction. The input signal switches the external head group of a synthetic signal transducer embedded in the membrane from polar (blue) to apolar (purple), allowing it to translocate through the membrane. Binding of a charged co-factor to the inner head group (red) activates the catalyst (green). (b) Catalytic surfactant generation. The catalyst hydrolyses a substrate (grey) generating a surfactant (yellow). (c) Cargo release. The surfactant enhances the permeability of the membrane to polar solutes, facilitating cargo release (pink). (d) Molecular structures of protonated signal transducer $1 \cdot \mathrm{H}^{+}(\mathrm{OFF}$ state) and the activated catalyst $1 \cdot \mathrm{Zn}^{2+}(\mathrm{ON}$ state).

The first stage of the process is transduction of the chemical signal across the lipid bilayer membrane by the synthetic transducer (Fig 1.a). ${ }^{16,17}$ The transducer is shorter than the width of the bilayer, so when the external recognition head group is in a charged state, it preferentially sits in the aqueous phase, and the transducer is located in the outer leaflet. The input signal (the first messenger, orange) switches the external head group to an apolar state, allowing the transducer to translocate through the membrane. When the internal head group binds a charged co-factor, it preferentially sits in the aqueous phase, fixing the transducer in the inner leaflet. In the second step (Fig. 1b), the activated catalyst hydrolyses the encapsulated substrate (grey) to generate the internal second messenger, a surfactant (yellow). In the final stage, the hydrophobic surfactant embeds in the lipid bilayer membrane, rendering it permeable to the cargo molecules which diffuse out of the vesicle (Fig. 1c). In this work, we used the $\mathrm{pH}$-controlled signal transducer $\mathbf{1}^{16}$ to initiate intra-vesicle surfactant generation (Fig. 1d). Transducer 1 features a morpholine head group, which is protonated at neutral $\mathrm{pH}\left(1 \cdot \mathrm{H}^{+}\right)$and can be deprotonated by raising the $\mathrm{pH}$ on the outside of the vesicle to initiate translocation. The pro-catalyst head group is a neutral pyridine oxime, which becomes a catalyst for ester hydrolysis when it binds zinc $\left(1 \cdot \mathrm{Zn}^{+}\right)$.

\section{RESULTS AND DISCUSSION}

The triggered release mechanism shown in Figure 1 involves the cooperation of multiple functional components: (a) transmembrane signal transduction, (b) catalytic surfactant generation and (c) cargo release. To address this complexity, we investigated the behaviour of each component individually, prior to assembling the complete functional system.

Cargo Release Experiments. Identification of a suitable surfactant that could be generated using the signal transduction system was addressed first. In the $\mathrm{ON}$ state of the transducer $\left(1 \cdot \mathrm{Zn}^{+}\right)$, the zinc-oxime complex catalyses the hydrolysis of ester substrates, so the catalyst could be used to generate a hydrophobic carboxylic acid as the second messenger surfactant product. We identified 2-naphthoic acid as a potential second messenger (compound 2, Fig. 2). This compound is a known hydrotrope ${ }^{29}$ and membrane permeable. ${ }^{30}$ The surfactant properties were assessed using calcein release experiments. Calcein is a membraneimpermeable fluorophore (Fig. 2) that self-quenches at high concentrations. When calcein is loaded inside vesicles at a concentration of $70 \mathrm{mM}$ self-quenching occurs. Any calcein efflux from the vesicles results in fluorophore dilution and reduction in the extent of self-quenching, which is detected by an increase in the calcein emission. Calcein fluorescence can therefore be used as an indicator for lipid vesicle leakage. ${ }^{31}$ The extent of calcein release can be quantified by lysis of the vesicles with detergent (Triton X-100) allowing the change in fluorescence emission intensity to be calibrated relative to release of the entire contents of the vesicles.

Vesicles containing a self-quenched calcein solution were assembled by hydrating a dried mixture of 1,2-dioleoyl-sn-glycero-3phosphocholine (DOPC) and 1,2-dioleoyl-sn-glycero-3phosphoethanolamine (DOPE) lipids in an aqueous solution containing $70 \mathrm{mM}$ calcein in HEPES buffer at $\mathrm{pH}$ 7. The suspension was extruded ${ }^{32}$ through a polycarbonate membrane to afford vesicles of an average diameter of around $200 \mathrm{~nm}$. The vesicles were separated from the external solution using size exclusion chromatography and then suspended in a non-buffered sodium chloride solution ( $\mathrm{pH} 7$ ). An aliquot of a solution of 2 in methanol was added to the vesicle suspension, and rapid release of calcein from the vesicles was observed (black data, Fig. 2b). In the absence of 2 (blue data) or upon addition of methanol (grey data), the calcein remained encapsulated, and the vesicles were stable until the detergent was added. ${ }^{33}$ These experiments demonstrate that 2 has suitable surfactant properties to act as the second messenger illustrated in Figure 1.

Catalytic Surfactant Generation. Having identified naphthoic acid 2 as a suitable second messenger for triggering cargo release, we turned our attention to generating this surfactant in-situ from an ester precursor encapsulated inside vesicles (Fig. 3). The ester substrate for the hydrolysis reaction, 3, was prepared in one 
step from 4 and 2-naphthoyl chloride (see ESI). Substrate 3 is water soluble, highly charged, membrane impermeable and nonfluorescent. Cleavage of the ester reveals fluorescent pyranine 4 (8hydroxypyrene-1,3,6-trisulfonate), which enables convenient monitoring of the reaction and quantification of the production of surfactant 2 .

(a)

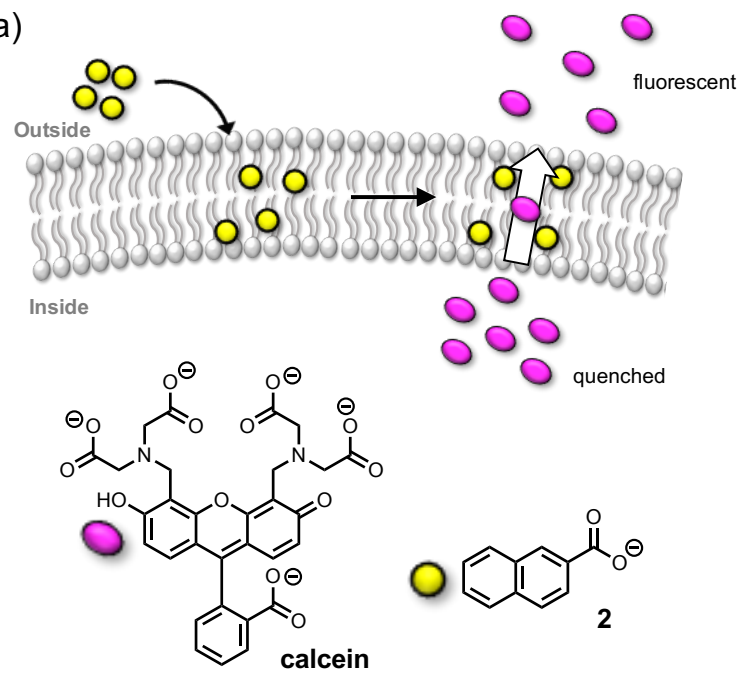

(b)

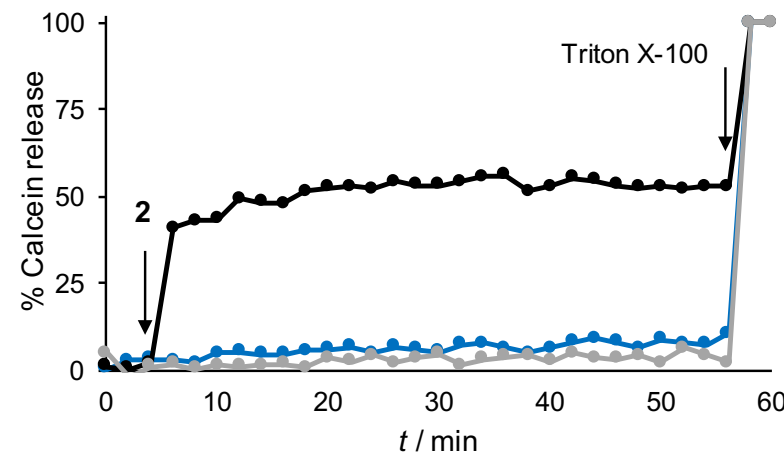

Figure 2. Calcein release assay. (a) Compound 2 (yellow) was added externally to $200 \mathrm{~nm}$ DOPC/DOPE vesicles loaded internally with 70 $\mathrm{mM}$ calcein (pink). The vesicles (lipid concentration $0.5 \mathrm{mM}$ ) contained $250 \mathrm{mM}$ HEPES at $\mathrm{pH} 7$ and were suspended in $400 \mathrm{mM} \mathrm{NaCl}$. (b) Calcein release measured by fluorescence emission at $540 \mathrm{~nm}(\mathrm{ex}-$ citing at $470 \mathrm{~nm}$ ). Blue data: background leakage of calcein. Grey data: background leakage of calcein in the presence of $4 \mu \mathrm{L}$ methanol. Black data: release of calcein triggered by addition of 2 in $4 \mu \mathrm{L}$ methanol (final concentration $50 \mu \mathrm{M}$ ). Calcein release was calibrated to $100 \%$ release by lysis with Triton $\mathrm{X}-100$.

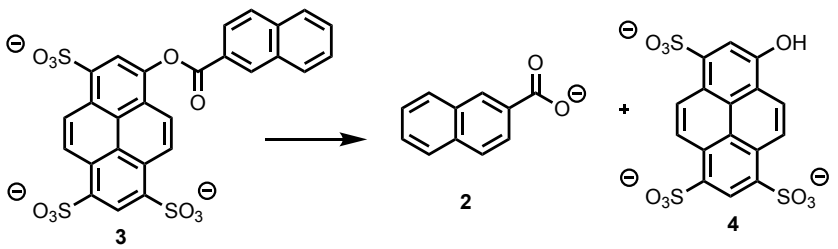

Figure 3. Molecular structures of ester substrate 3 and hydrolysis products, surfactant 2 and fluorophore 4.
Signal transducer 1 was synthesised as previously described. ${ }^{16}$ The functional vesicles were assembled by hydrating a dried mixture of lipids and transducer 1 in an aqueous solution containing substrate 3, zinc chloride and HEPES buffer at $\mathrm{pH} 7$. The suspension was extruded, the vesicles were separated using size exclusion chromatography and then suspended in sodium chloride solution to afford final bulk concentrations of $2 \mathrm{mM}$ lipids and $50 \mu \mathrm{M}$ transducer. A control system was prepared in the same way, but in the absence of transducer 1. Incorporation of the transducer during vesicle preparation results in a statistical distribution of transducer 1 in the inner and outer leaflets of the membrane. However, only the molecules in the outer leaflet are in the correct orientation to interact with the external signal and to catalyse reactions inside the vesicle. The remaining $50 \%$ of the transducers that are in an inverted orientation are not sensitive to changes in external $\mathrm{pH}$ and cannot catalyse reactions inside the vesicle, so they are silent in the experiments.

Figure 4 shows a signal transduction experiment with substrate 3 . Sodium hydroxide was added to the external vesicle solution to initiate translocation of 1 across the membrane, and the fluorescence emission due to formation of $\mathbf{4}$ was measured.

(a)

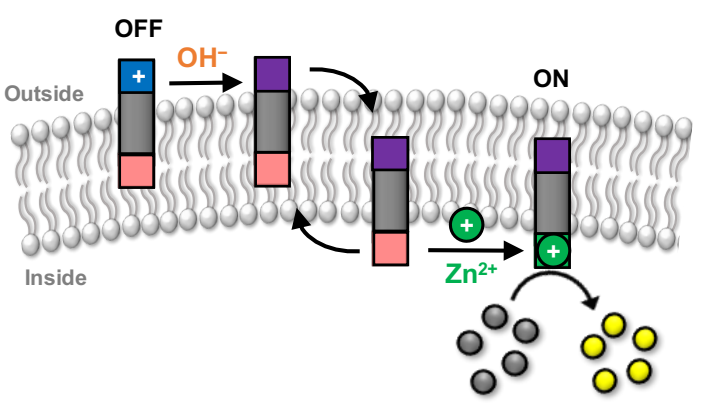

(b)

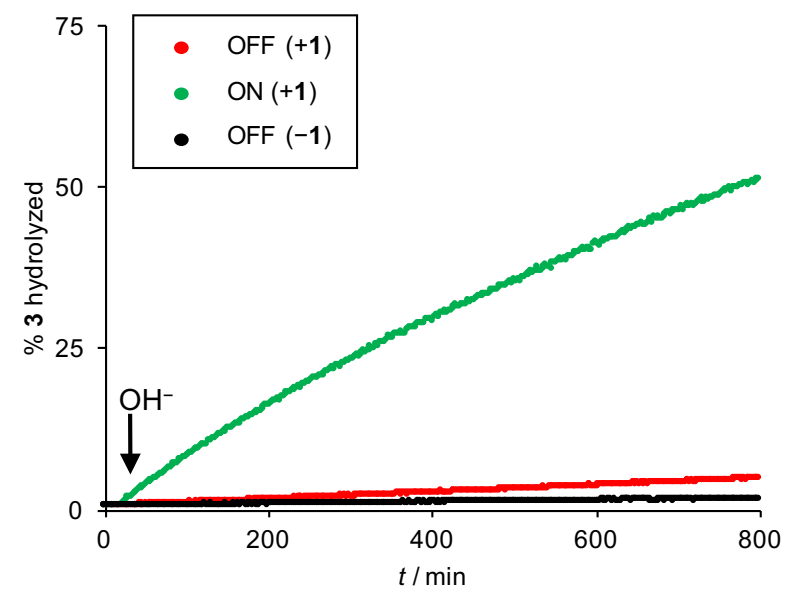

Figure 4. (a) Surfactant generation inside vesicles controlled by transmembrane signal transduction. Activation of transducer 1 by extra-vesicle hydroxide leads to catalysed hydrolysis of $\mathbf{3}$ (grey) inside the vesicles to generate surfactant 2 (yellow), which is quantified by measuring the fluorescence emission arising from formation of fluorophore 4. (b) Time dependence of the normalized fluorescence emission intensity at $510 \mathrm{~nm}$ (exciting at $415 \mathrm{~nm}$ ) of vesicles in the presence (red data) and absence (black data) of transducer 1, and following $\mathrm{NaOH}$ addition (green data) to initiate signal transduction. All experiments were conducted in $200 \mathrm{~nm}$ DOPC/DOPE vesicles $(2 \mathrm{mM}$ final lipid concentration) containing $250 \mu \mathrm{M}$ ester 3, $250 \mu \mathrm{M} \mathrm{ZnCl}_{2}$ and $250 \mathrm{mM}$ HEPES buffer at $\mathrm{pH} 7$, and suspended in $250 \mathrm{mM} \mathrm{NaCl}$. The loading of transducer 1 was $2.5 \mathrm{~mol} \%$. 
In the OFF state at $\mathrm{pH} 7$, conversion of 3 to 4 was minimal (red data, $<4 \%$ after $800 \mathrm{mins}$ ), and comparable to the background solvolysis rate in the absence of transducer 1 (black data). At $\mathrm{pH} 7$, the external head-group of the transducer is protonated, trapping it in the outer leaflet, such that the pyridine oxime head-group is embedded inside the membrane in an inactive state. Addition of an aliquot of base to the suspension instantaneously raised the external $\mathrm{pH}$ to 9 (determined using a $\mathrm{pH}$-electrode) and led to a sharp increase in fluorescence intensity due to formation of 4 . At $\mathrm{pH} 9$, the morpholine head group is deprotonated, so transducer 1 can translocate across the bilayer, generating the $\mathrm{ON}$ state in which the zinc-pyridine oxime complex catalyses the hydrolysis of $\mathbf{3}$ inside the vesicles (green data, Fig. 4). These experiments demonstrate that ester 3 is a suitable substrate for the signal transduction system and that surfactant 2 can be generated in response to an external $\mathrm{pH}$ signal.

The experiments also provide some insight into how surfactant 2 generated by the in-situ hydrolysis reaction affects the integrity of the vesicle membranes. The other product of the hydrolysis reaction 4 acts as a fluorescent sensor for local $\mathrm{pH}^{34}$ In the experiments described above, vesicles were prepared with an internal $\mathrm{pH}$ of 7 , and in the OFF state, this $\mathrm{pH}$ was maintained. However, in the ON state, the apparent value of the internal $\mathrm{pH}$ increased with time, reaching a value of around $\mathrm{pH} 8$ after 60 minutes (Fig. 5). ${ }^{35}$

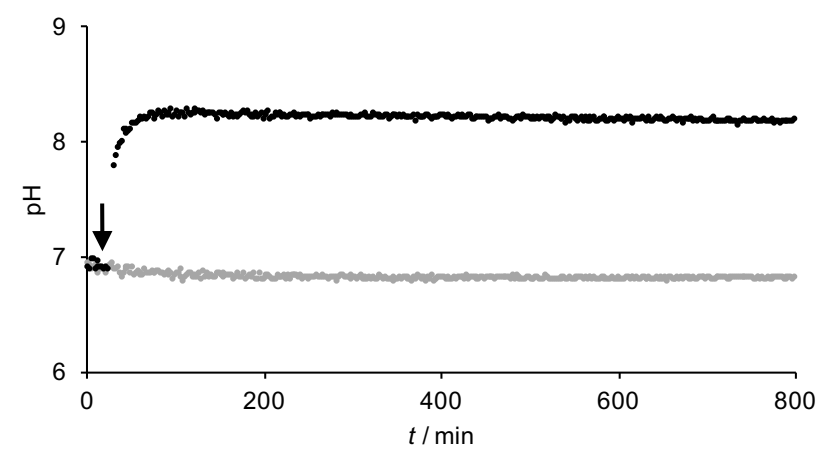

Figure 5. Effect of transmembrane signal transduction on the apparent local $\mathrm{pH}$ determined spectroscopically by ratiometric analysis of the fluorescence emission from 4 (experimental conditions as in Fig. 4). Grey data: vesicles incubated at $\mathrm{pH} 7$ (OFF state). Black data: vesicles incubated at $\mathrm{pH} 7$, then $\mathrm{pH} 9$ (arrow indicates addition of hydroxide to form the ON state).

This observation can be rationalised in two ways. One explanation is that 2-naphthoic acid $\mathbf{2}$ generated by the catalysed hydrolysis of 3 enhances the permeability of the membrane with respect to $\mathrm{H}^{+} / \mathrm{OH}$. Since the external $\mathrm{pH}$ is 9 and the internal $\mathrm{pH}$ is 7 , equilibration of the proton gradient across the membrane would lead to an increase of $\mathrm{pH}$ on the inside of the vesicles, where it is reported by encapsulated 4 . The alterative explanation is that 2 causes the membrane to become permeable to 4 which diffuses out of the vesicles. In this case, the apparent $\mathrm{pH}$ is a population-weighted average value determined by the amount of 4 inside and outside of the vesicles. To distinguish these two scenarios, the integrity of the vesicles after the transmembrane signalling reaction was analysed by re-purifying the vesicle suspension using size exclusion chromatography. This process removes any small molecules that are not encapsulated inside vesicles. After purification, a significant reduction in the intensity of the fluorescence emission arising from 4 was observed (Fig. 6), demonstrating that formation of surfactant 2 leads to leakage of 4 from the vesicles.

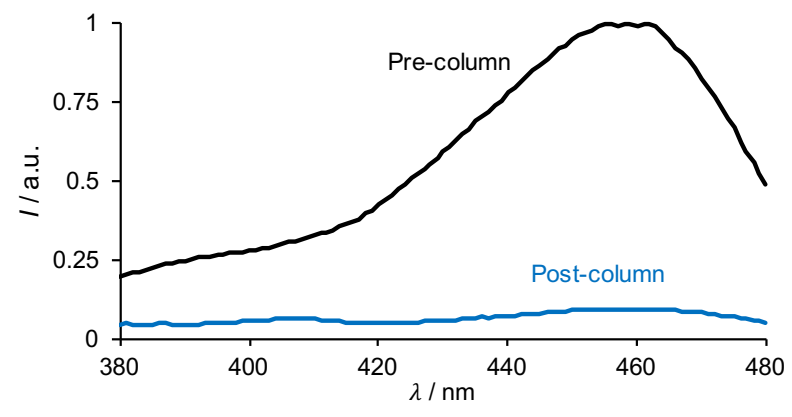

Figure 6. Fluorescence excitation spectrum (emission at $510 \mathrm{~nm}$ ) of vesicles at the end of a signal transduction experiment (ON state after 1000 min, experimental conditions as in Fig. 4). Spectra were recorded before (black data) and after (blue data) SEC purification to remove extra-vesicle material and normalized to account for dilution during purification.

Transmembrane Signal Transduction Triggered Release Experiments. Having established that the individual components of the system function separately, calcein release initiated by in-situ generation of 2 by transmembrane signal transduction was investigated.

(a)

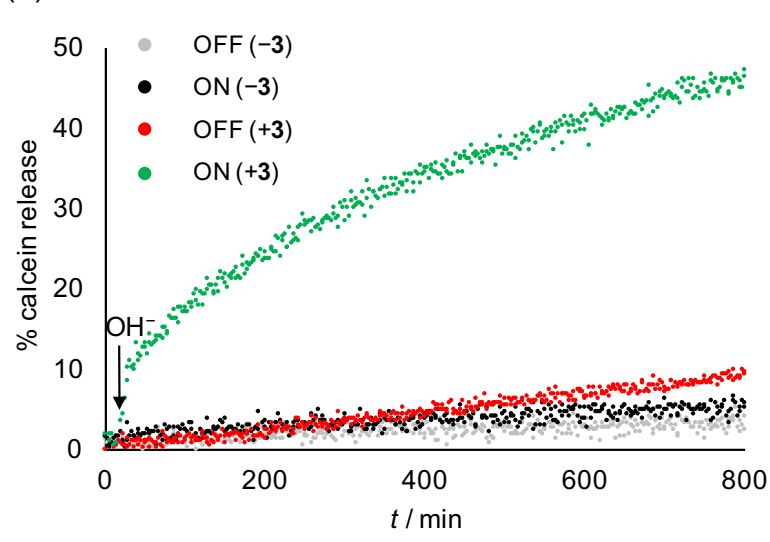

Figure 7. (a) Catalysed surfactant generation inside vesicles initiated by transmembrane signal transduction leads to release of encapsulated cargo by permeabilization of the membrane. (b) Time dependence of calcein from $200 \mathrm{~nm}$ DOPC/DOPE vesicles (lipid concentration 0.5 $\mathrm{mM}$ ) containing $70 \mathrm{mM}$ calcein, $250 \mu \mathrm{M} \mathrm{ZnCl} 2$ and $250 \mathrm{mM}$ HEPES at $\mathrm{pH} 7$ suspended in $400 \mathrm{mM} \mathrm{NaCl}$, in the presence $(+3)$ and absence $(-3)$ of encapsulated substrate $3(25 \mu \mathrm{M})$. The transducer was switched from the OFF to the ON state by addition of $\mathrm{NaOH}$ (arrow). Calcein emission at $540 \mathrm{~nm}$ (exciting at $470 \mathrm{~nm}$ ) was calibrated to $100 \%$ release by lysis with Triton X-100. 
Vesicles containing membrane-embedded transducer 1 and encapsulating $70 \mathrm{mM}$ calcein, $25 \mu \mathrm{M} 3$ and $250 \mu \mathrm{M} \mathrm{ZnCl}_{2}$ were prepared. The results of the triggered release experiments are shown in Figure 7. Addition of hydroxide to switch the transducer to the $\mathrm{ON}$ state led to rapid release of calcein from the vesicles only when the ester substrate was also present inside the vesicles (green data in Figure $7 \mathrm{~b}$ ). This shows that the three stages of the process (signal transduction, catalytic generation of surfactant and cargo release) can cooperate in the fully functional controlled release mechanism, facilitating cargo release from inside vesicles in response to an extra-vesicle signal. After 800 minutes, the amount of calcein released is 15 times greater than the amount of hydroxide added to initiate signalling. The observed signal amplification is due to catalysis of production of the second messenger, which is itself a catalyst for transport of the output signal across the lipid membrane. Such catalytic cascade processes are one of the hallmarks of signal amplification in biological systems.

To determine whether cargo release occurs by complete lysis of the vesicles or by permeabilisation of intact vesicles, size exclusion chromatography was used to re-isolate the vesicles at the end of the experiment. Analysis of the purified vesicles by dynamic light scattering (DLS) showed that the size distribution of the vesicles obtained after calcein release (ON state) was identical to that of the precursors in the OFF state (see ESI). ${ }^{1} \mathrm{H}$ NMR experiments show that the total concentration of phospholipid present in vesicles was unchanged in the re-isolated vesicles after calcein release. ${ }^{36}$ Purified vesicles from the $\mathrm{ON}$ and OFF experiments were separately dissolved in a mixture of chloroform and methanol containing an internal standard (3-(trimethylsilyl)propionic-2,2,3,3- $\mathrm{d}_{4}$ acid sodium salt). Integration of the signal due to the terminal methyl group of the phospholipid was used to determine the lipid concentration by reference to the integral of the signal due to the internal standard (see ESI). The results were identical for the vesicles obtained from the $\mathrm{ON}$ and the OFF experiments. These experiments show that the vesicles were still intact after cargo release, so 2 triggers the release of calcein by destabilisation of the membrane at subsolubilising concentrations.

\section{CONCLUSIONS}

The controlled generation of surfactant molecules from a precursor encapsulated inside a lipid bilayer vesicle has been used to trigger the release of hydrophilic molecules co-encapsulated in the interior. An extra-vesicle input signal ( $\mathrm{pH}$ change) was used to activate a membrane-bound artificial signal transducer, which catalyzed the intra-vesicle generation of a surfactant (2-naphthoic acid). Permeabilisation of the membrane at sub-solubilizing surfactant concentration led to cargo efflux, as demonstrated by a calcein release assay. These results suggest that combining artificial signal transducers that can be activated by a range of input signals, with controlled surfactant synthesis inside vesicles, may provide a generic platform with which to develop triggered release applications such as for drug delivery.

\section{ASSOCIATED CONTENT}

\section{Supporting Information}

The Supporting Information is available free of charge on the ACS Publications website at DOI: $10.1039 / \mathrm{x} 0 \mathrm{xx} 00000 \mathrm{x}$.

Synthetic procedures, characterization, and additional experimental details (PDF)

\section{AUTHOR INFORMATION}

\section{Corresponding Author}

herchelsmith.orgchem@ch.cam.ac.uk; n.h.williams@sheffield.ac.uk

\section{ORCID}

Matthew J. Langton: 0000-0003-1555-3479

Christopher A. Hunter: 0000-0002-5182-1859

Nicholas H. Williams: 0000-0002-4457-422

\section{Notes}

The authors declare no competing financial interests.

\section{ACKNOWLEDGMENT}

We thank the University of Cambridge for an Oppenheimer Research Fund for an Early Career Research Fellowship (M.J.L).

\section{REFERENCES}

(1) Krauss, G. Biochemistry of Signal Transduction and Regulation, 3rd ed.; Wiley: New York, 2006.

(2) Simon, M. I.; Strathmann, M. P.; Gautam, N. Science 1991, 252, 802-808.

(3) Bennett, I. M.; Farfano, H. M. V.; Bogani, F.; Primak, A.; Liddell, P. A.; Otero, L.; Sereno, L.; Silber, J. J.; Moore, A. L.; Moore, T. A.; Gust, D. Nature 2002, 420, 398-401.

(4) Fyles, T. M. Chem. Soc. Rev. 2007, 36, 335-347.

(5) Davis, J. T.; Okunola, O.; Quesada, R. Chem. Soc. Rev. 2010, 39, 3843-3862.

(6) Matile, S.; Jentzsch, A. V.; Montenegro, J.; Fin, A. Chem. Soc. Rev. $2011,40,2453-2474$.

(7) Vargas Jentzsch, A.; Hennig, A.; Mareda, J.; Matile, S. Acc. Chem. Res. 2013, 46, 2791-2800

(8) Stanzl, E. G.; Trantow, B. M.; Vargas, J. R.; Wender, P. A. Acc. Chem. Res. 2013, 46, 2944-2954.

(9) Gale, P. A.; Davis, J. T.; Quesada, R. Chem. Soc. Rev. 2017, 46, 2497-2519

(10) Barton, P.; Hunter, C. A.; Potter, T. J.; Webb, S. J.; Williams, N. H. Angew. Chem. Int. Ed. 2002, 41, 3878-3881.

(11) Dijkstra, H. P.; Hutchinson, J. J.; Hunter, C. A.; Qin, H.; Tomas, S.; Webb, S. J.; Williams, N. H. Chem. - Eur. J. 2007, 13, 72157222.

(12) Bernitzki, K.; Schrader, T. Angew. Chem. Int. Ed. 2009, 48, 8001-8005.

(13) Bernitzki, K.; Maue, M.; Schrader, T. Chem. - Eur. J. 2012, 18 13412-13417.

(14) De Poli, M.; Zawodny, W.; Quinonero, O.; Lorch, M.; Webb, S. J.; Clayden, J. Science 2016, 352, 575-580.

(15) Lister, F. G. A.; Le Bailly, B. A. F.; Webb, S. J.; Clayden, J. Nat. Chem. 2017, 9, 420-425.

(16) Langton, M. J.; Keymeulen, F.; Ciaccia, M.; Williams, N. H.; Hunter, C. A. Nat. Chem. 2017, 9, 426-430.

(17) Langton, M. J.; Williams, N. H.; Hunter, C. A. J. Am. Chem. Soc. 2017, 139, 6461-6466

(18) Striggow, F.; Ehrlich, B. E. Curr. Opin. Cell Biol. 1996, 8, 490495.

(19) Torchilin, V. P. Nat. Rev. Drug Discov. 2005, 4, 145-160.

(20) Allen, T. M.; Cullis, P. R. Adv. Drug Deliv. Rev. 2013, 65, 36-48.

(21) Mantripragada, S. Prog. Lipid Res. 2002, 41, 392-406.

(22) Barenholz, Y. (Chezy). J. Controlled Release 2012, 160 , $117-$ 134.

(23) Pattni, B. S.; Chupin, V. V.; Torchilin, V. P. Chem. Rev. 2015, 115, 10938-10966.

(24) Ruiz, J.; Goñi, F. M.; Alonso, A. Biochim. Biophys. Acta BBA Biomembr. 1988, 937, 127-134. 
(25) Lasch, J. Biochim. Biophys. Acta BBA - Rev. Biomembr. 1995, 1241, 269-292.

(26) Lichtenberg, D.; Ahyayauch, H.; Goñi, F. M. Biophys. J. 2013, 105, 289-299.

(27) Ahyayauch, H.; Bennouna, M.; Alonso, A.; Goñi, F. M. Langmuir 2010, 26, 7307-7313.

(28) Stano, P.; Luisi, P. L. Chem. Commun. 2010, 46, 3639-3653.

(29) Atwood, D. Surfactant Systems: Their chemistry, pharmacy and biology; Springer, 2012.

(30) Xiang, T.-X.; Anderson, B. D. J. Membr. Biol. 1994, 140, 111122.

(31) Matile, S.; Sakai, N. In Analytical Methods in Supramolecular Chemistry; Schalley, C. A., Ed.; Wiley, 2012; pp 711-742.

(32) MacDonald, R. C.; MacDonald, R. I.; Menco, B. P.; Takeshita, K.; Subbarao, N. K.; Hu, L. R. Biochim. Biophys. Acta 1991, 1061, 297-303.

(33) Compound 2 is not very soluble in water, and precipitation was observed upon addition of the methanol solution of 2 to the aqueous vesicle suspension. Quantitative release of calcein was therefore not achieved by a single addition.
(34) Clement, N. R.; Gould, J. M. Biochemistry (Mosc.) 1981, 20, 1534-1538.

(35) Control experiments with vesicles containing 3 at $\mathrm{pH} 7$ and at $\mathrm{pH}$ 8 show that this change in $\mathrm{pH}$ has no significant effect on the background solvolysis rate (see ESI). For signal transduction systems using an acetyl-derivative of $\mathbf{3}$ as the substrate (ref. 16), the intra-vesicle $\mathrm{pH}$ remained stable during signal transduction experiments, which implies that the change in $\mathrm{pH}$ reported here is due to formation of $\mathbf{2}$.

(36) Hein, R.; B. Uzundal, C.; Hennig, A. Org. Biomol. Chem. 2016, $14,2182-2185$.

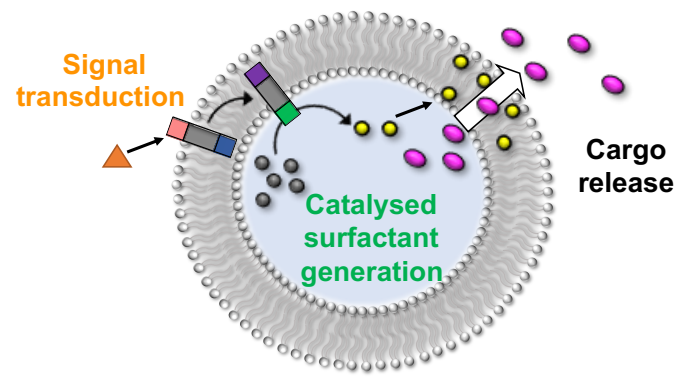

